\title{
A case of recurrent re-admissions with severe hyperemesis Gravidarum
}

\author{
P.Jinadev', K. Jacob² Department of Diabetes \& Endocrinology \\ ${ }^{1}$ Central Manchester University Hospitals, M13 0JE. \\ 2Pilgrim Hospital, United Lincolnshire Trust, Boston, PE21 9QS
}

\section{Case presentation}

- Mrs XY, 29 years old,(gravida 3,para 2), was admitted under the obstetrician with severe hyperemesis at 8 weeks gestation. She had 3 admissions prior to this admission with similar complaint.

- She was treated with IV fluids, Ondansetron, Thiamine at all admissions.

\section{Past History}

- Autoimmune primary hypothyroidism

- Depression

\section{Medications}

- Levothyroxine 125 mcg once daily

- Sertraline

\section{Endocrinology referral}

- Endocrinology opinion was requested by the obstetrician for review of deranged TFTs

\section{Clinical examination}

- MRS XY was found to be hyper pigmented especially at elbows and buccal mucosa

- She was dehydrated. Her BP 93/52 mm Hg.

- She was emotionally distressed (expressed the desire to terminate her pregnancy).

\section{Investigations}

- S.Sodium 136 mmol/l( 134-146)

- S. Potassium 3.8mmol// (3.5 - 5.3)

- Urea $0.5 \mathrm{mmol} / \mathrm{l}(2.5-5.8)$

- Random glucose $7.9 \mathrm{mmol} / \mathrm{l}(3-6)$

- Hb 10.4g/l, MCV 87 fl, eosinophils normal

- Tissue transglutaminase antibodies IgA negative.

\section{Endocrinology tests}

- TSH 0.24 mu/l (0.35 - 4.94), FT 15.8 $\mathrm{pmol} / \mathrm{l}(9-19.1)$

- S.Cortisol and ACTH

\begin{tabular}{|c|c|}
\hline \multicolumn{2}{|c|}{ Short Synacten test } \\
\hline Basal Cortisol & $<20 \mathrm{nmol} / \mathrm{l}$ \\
\hline 30 min Cortisol & $<20 \mathrm{nmol} / \mathrm{l}$ \\
\hline
\end{tabular}

- ACTH elevated at> 1250ng/l.

- Adrenal antibodies positive.

- Plasma renin activity $2.4 \mathrm{nmol} / \mathrm{hr} / \mathrm{l}$

\section{Diagnosis and Treatment}

- Diagnosis of Addisonian crisis was made.

- She was immediately treated with IV Hydrocortisone 100mg followed by $100 \mathrm{mg}$ 6th hourly IM for 48 hours and IV fluids

\section{Outcome and Follow up}

- There was no further admissions during pregnancy.

- She delivered a healthy baby at 38 weeks gestation.

- The pigmentation had improved.

- Her medications consist of Hydrocortisone $10 \mathrm{mg} / 5 \mathrm{mg} / 5 \mathrm{mg}$, Fludrocortisone $100 \mathrm{mcg}$ once daily and Thyroxine $125 \mathrm{mcg}$ once daily.

\section{Discussion}

- Addison's disease in pregnancy can be easily missed due to similar presentation as in early pregnancy. (vomiting, fatigue, hyperpigmentation and low blood).

- This lady also had autoimmune hypothyroidism which can be associated with autoimmune adrenal insufficiency especially as part of polyglandular autoimmune syndrome Type 2.

- Addison's disease developing in pregnancy may result in adrenal crisis if delay in diagnosis.

Prior to the availability of glucocorticoids, hypoadrenalism was associated with significant mortality with almost $80 \%$ dying within 2 years.(1)

- Hyperpigmention in pregnancy can be another source of confusion.

Hyper pigmentation usually occurs in discrete localised areas including around areola,nipples, axillae and genitalia.

- Melasma in pregnancy typically affects the sun exposed areas of the face.

- The hyperpigmentation of chronic primary hypoadrenalism is usually generalised but also noticed on the mucosa, areas of frictions and old scars

- In our patient the hyperpigmentation was considered due to pregnancy until reviewed by the endocrinologist on the ward.

\section{Conclusion}

- Our patient with known autoimmune hypothyroidism presented with severe hyperemesis gravidarum and hyperpigmentation in her 3rd pregnancy.

She was 8 weeks pregnant. She did not these problems with her previous pregnancies. A high index of suspicion for adrenal insufficiency is necessary especially if other autoimmune conditions coexist.

\section{Reference}

Dunlop D - 86 cases of Addison's Disease. British Medical Journal 1963;2:887 